\title{
Differentiation of human adipose tissue SVF cells into cardiomyocytes
}

\author{
Agnieszka Sliwa $\cdot$ A. Balwierz $\cdot$ B. Kiec-Wilk $\cdot$ \\ A. Polus · A. Knapp · A. Dembinska-Kiec
}

Received: 15 May 2009/Accepted: 27 May 2009/Published online: 16 June 2009

(C) Springer-Verlag 2009

\begin{abstract}
Progenitor cells have been extensively studied and therapeutically applied in tissue reconstructive therapy. Stromal vascular fraction (SVF) cells, which are derived from adipose tissue, may represent a potential source of the cells which undergo phenotypical differentiation into many lineages both in vitro as well as in vivo. The goal of this study was to check whether human SVF cells may differentiate into cardiomyocyte-like entities. Human SVF cells were induced to differentiate by their incubation in Methocult medium in the presence of SCF, IL-3 and IL-6. Morphological transformation of the cells was monitored using optical light microscope, whereas changes in expression of the genes typical for cardiac phenotype were measured by qRT-PCR. Incubation of the human SVF cells in the medium that promotes cardiomyocyte differentiation in vitro resulted in formation of myotubule-like structures accompanied by up-regulation of the myocardium-characteristic genes, such as GATA, MEF2C, MYOD1, but not ANP. Human SVF cells differentiate into cardiomyocyte-
\end{abstract}

\footnotetext{
A. Sliwa - A. Balwierz - B. Kiec-Wilk - A. Polus - A. Knapp ·

A. Dembinska-Kiec $(\bowtie)$

Department of Clinical Biochemistry,

Collegium Medicum, Jagiellonian University,

15a Kopernika Str., 31-501 Krakow, Poland

e-mail: mbkiec@cyf-kr.edu.pl

A. Sliwa

e-mail: agasliw@interia.pl

A. Balwierz

Postgraduate School of Molecular Medicine,

61 Żwirki i Wigury Str., 02-091 Warsaw, Poland
}

like cells in the presence of the certain set of myogenesis promoting cytokines.

Keywords SVF Adipose tissue $\cdot$ Cardiomyocytes

\section{Introduction}

Adipose tissue is an active endocrine organ that secretes adipokines along with a number of pro-angiogenic cytokines and growth promoting factors (reviewed in [12]). It has been found that human adipose tissue contains a population of non-characterized cells, which are capable to undergo adipogenic, osteogenic, chondrogenic or myogenic differentiation in vitro as well as in vivo. These cells called collectively stromal vascular fraction (SVF) demonstrate multilineage potential and extensive self-renewal capacity [14].

Stem cells have been vigorously investigated as critical component of reconstructive therapies for a variety of heart diseases [4] since they are capable to differentiate into functional cardiomyocytes [5]. The adipose tissue SVF cells were suggested as an alternative source of progenitor cells for autologous application since known sources of such cells suitable for therapeutic usage are limited. SVF cells are already used for reparation of tendon, bone as well as skeletal muscle tissue in humans $[1,6,10]$. It has been also demonstrated that functional cardiomyocyte-like cells could be derived directly from mouse adipose tissue when incubated with certain cytokines such as stem cell factor (SCF), interleukin-3 (IL-3) and interleukin-6 (IL-6) [6, 10].

The aim of this study was to investigate the possible differentiation of the human adipose tissue-derived SVF cells into cardiomyocytes. 


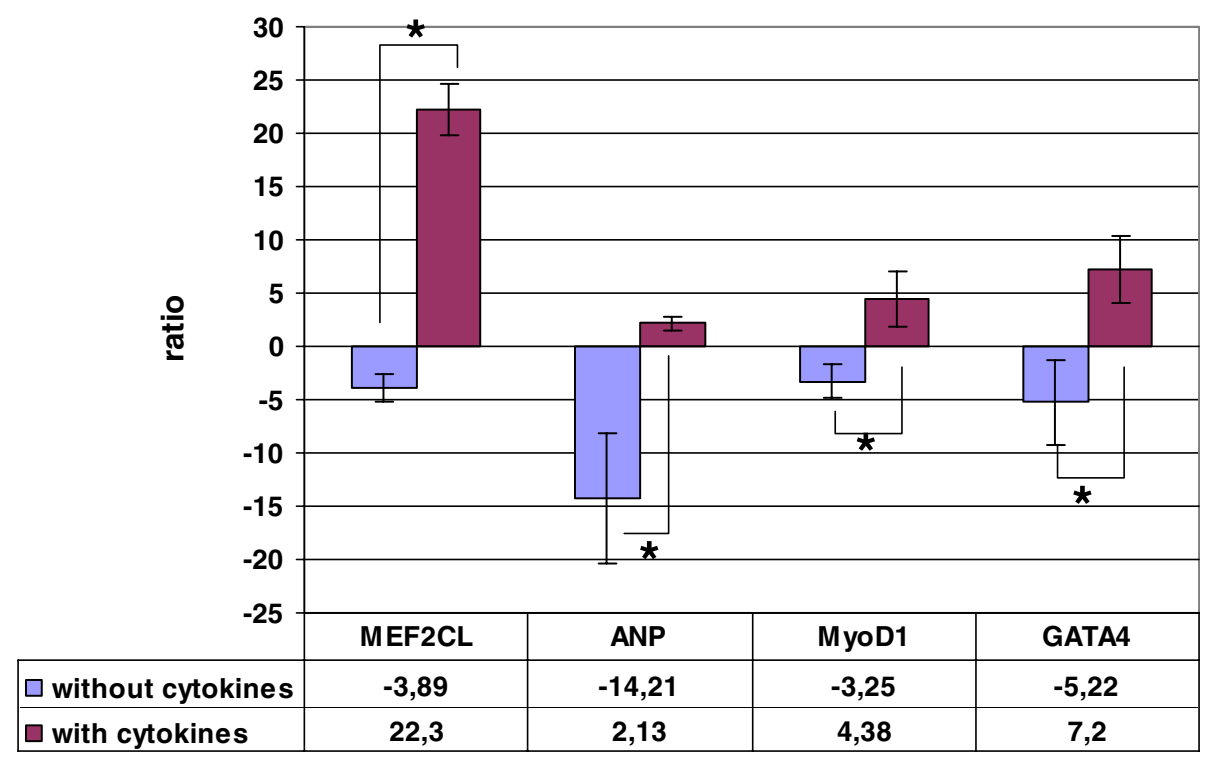

Fig. 1 Changes in expression of typical for cardiomyogenesis genes in human SVF cells cultured in presence or absence of selected cytokines. 24-day incubation of freshly isolated human SVF cells in Methocult medium supplemented with SCF (50 ng/ml), IL-3 (10 ng/ $\mathrm{ml})$ and IL-6 (10 $\mathrm{ng} / \mathrm{ml})$ was followed by total RNA isolation and RTPCR analysis of ANP, GATA4, MEF2C and MYOD1 gene

\section{Materials and methods}

\section{Materials}

All reagents for tissue culture were purchased from Sigma Aldrich, Germany, except for fetal bovine serum (FBS) from Gibco and media for progenitor cells differentiation (StemCell Technologies, Vancouver, Canada). Reagents for analyses of gene expression were purchased from Invitrogen Life Technologies and QIAGEN.

\section{Isolation, culturing and differentiation of SVF cells}

All the experiments were performed according to the permission of Polish Ethic Commission (nr KBET/56/B/ 2006). Each patient signed a consent form before being included into the study.

The method used for the SVF progenitor cells isolation was based on the previously published procedures [3]. Subcutaneous adipose tissue scraps (5-10 g) were obtained from female patients (age 35-55 years) undergoing planned surgery. Isolated SVF cells were re-suspended in PBS, counted and seeded at 7,000 cells $/ \mathrm{ml}$ in Methocult GF 3534 (StemCell Technologies, Vancouver, Canada), an adaptation medium (AM).

Control SVF cells were cultured in Isocove's MDM H4230, a basal medium (StemCell Technologies, Vancouver, Canada), supplemented with $1 \%$ methylcellulose $(4,000 \mathrm{cps}), 30 \%$ fetal bovine serum, $1 \%$ bovine expression. The SVF cells cultured in adaptation medium were used as controls. Results are presented as average \pm SEM from three separate experiments. Statistical significance was set at $P<0.05$. $S C F$ Stem cell factor, ANP atrial natriuretic peptide, GATA4 GATA binding protein 4, MEF2C myocyte enhancer factor 2C, MYODI myogenic differentiation 1

serum albumin, $100 \mu \mathrm{M}$ 2-mercaptoethanol and $2 \mathrm{mM}$ L-glutamine.

Differentiating SVF cells were cultured in Methocult GF 3534 medium (StemCell Technologies, Vancouver, Canada) in order to induce their transformation into cardiomyocytes. In some incubations, the basal $\mathrm{H} 4230$ medium

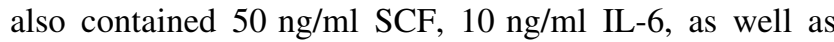
$10 \mathrm{ng} / \mathrm{ml}$ IL-3 [10].

Stromal vascular fraction cells, which were maintained in either adaptation or basal Methocult medium with or without cytokines listed above, were cultured for 24 days. Morphological changes in the cellular shape were monitored visually by optical light microscopy every 3 days. After 24 days of culture, cells were frozen with Trizol reagent for the myocardium-specific gene expression study [10].

\section{Measurement of gene expression}

Trizol method was used for isolation of total RNA from the SVF cells cultured in Methocult medium with or without cytokines. SVF cells cultured in adaptation medium (AM) were used as negative control.

RNA isolated from the SVF cells (1 $\mu \mathrm{g}$ total) was subjected to reverse transcription process with the use of a reverse transcription kit (Superscript) and random hexamers. The quantitative real-time PCR was performed with the QuantiTect SYBR Green PCR Kit (QIAGEN) using specific primers. 
Fig. 2 Phenotypic analysis of human SVF cells undergoing differentiation process. Optical light microscopic visualization of the SVF cells maintained in Methocult medium with or without SCF (50 ng/ml), IL-3 $(10 \mathrm{ng} / \mathrm{ml})$ and IL-6 $(10 \mathrm{ng} / \mathrm{ml})$ for 1,15 and 24 days. The cells were visualized at $\times 20$ enlargement

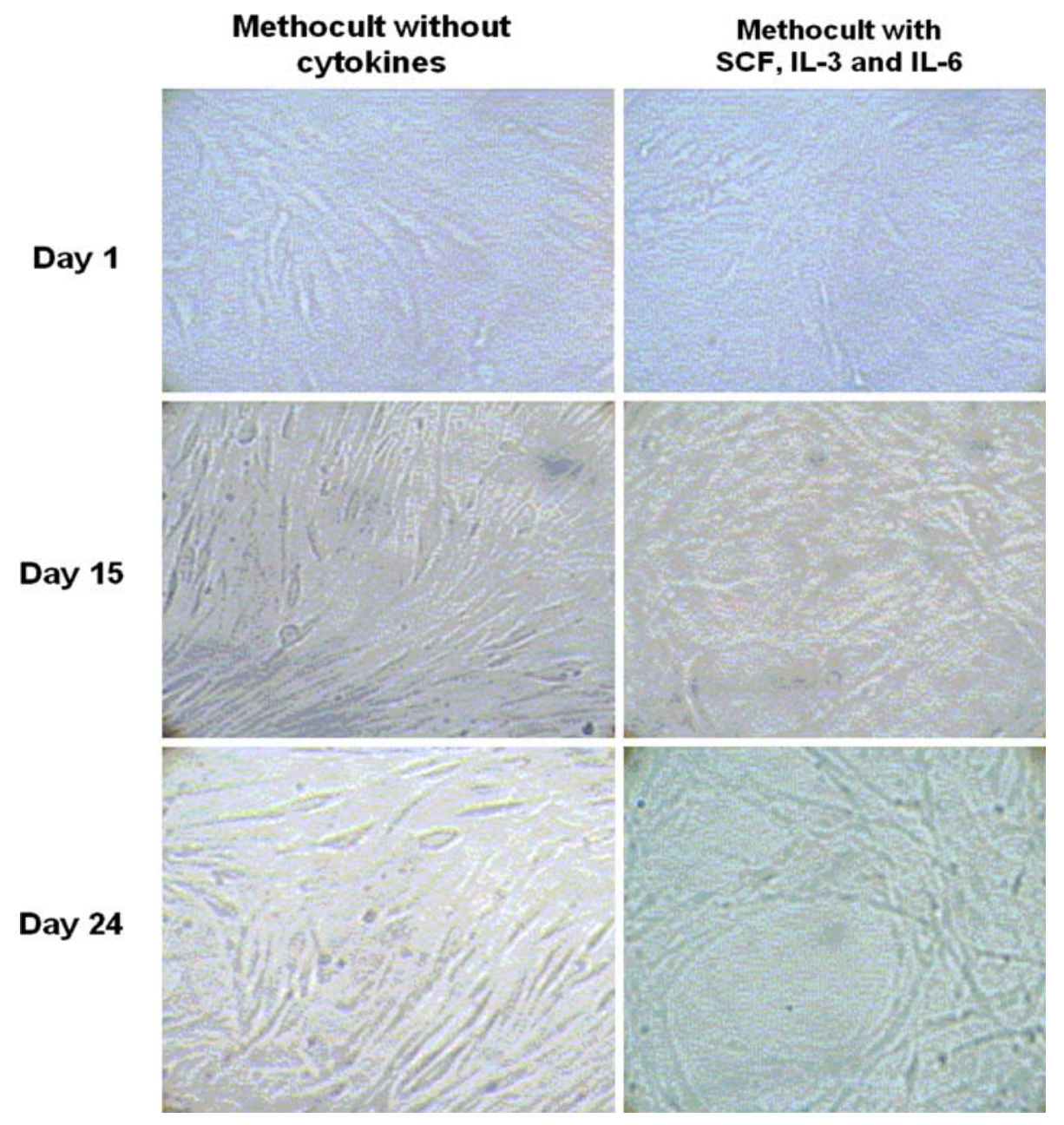

All the calculations were performed using calculation matrix for PCR efficiency program [9]. The expression rates were calculated as the normalized $C_{\mathrm{T}}$ (threshold cycle) difference between the sample and its corresponding control probe adjusted for their amplification efficiencies. Expression of the reference house-keeping GAPDH gene was used as a positive control.

\section{Statistical analysis}

Results of continuous variables' analysis were expressed as average \pm SEM collected from 3-5 experiments measured in triplicates. Normal distribution and homogeneity of variables were tested prior to further statistical analysis. Statistical correlations were calculated based on unpaired $t$ tests for comparisons of quantitative variables. Level of statistical significance was set at $P<0.05$ for the entire study. The statistical analyses were performed with Microsoft Excel (version 5.0, Microsoft Corporation, USA).

\section{Results}

Analysis of gene expression

Freshly isolated SVF cells were cultured in Methocult adaptation medium supplemented with selected cytokines in order to study potential differentiation of these progenitor cells into cardiomyocytes. Quantitative RT-PCR analysis of the total RNA derived from the SVF cells cultured in the presence of SCF, IL-3 and IL-6 demonstrated up-regulation of myocyte enhancer factor 2C (MEF), myogenic differentiation 1 (MyoD) and GATA binding protein 4 (GATA4). In contrast, same genes were down-regulated in the SVF cells cultured in the adaptation medium without the cardiomyogenesis-stimulating cytokines (Fig. 1). In addition, gene expression of another marker of cardiomyocytic phenotype, atrial natriuretic peptide (ANP), was significantly down-regulated (about 14 times) in the absence of SCF, IL-3 and IL- 6 in the culture medium. The level of ANP gene expression was 
normalized after supplementation with the above cytokines (Fig. 1). The presented results demonstrate that SVF cells cultured in Methocult with SCF, IL-3 and IL-6 express a set of genes characteristic for differentiated cardiomyocyte-like cells.

\section{Morphological changes}

Initial 24-h incubation of the SVF cells in Methocult medium with or without certain cytokines did not result in any morphological alterations (Fig. 2). Rates of cellular proliferation were similar in both Methocult media; however the shapes of those cells differed between the two groups. Starting on day 15th until the end of the experiment, day 24th, cells cultured in Methocult without SCF, IL-3 and IL-6 demonstrated typical fibroblast-like shapes, whereas cells cultured in the medium Methocult supplemented with above cytokines displayed differentiated features resembling myotubules, composed of aligned and branched myofibrils (Fig. 2). It is yet another verification of SVF potential to mature into cardiomyocyte population.

\section{Discussion}

The study presented above examines a hypothesis that human SVF cells are capable to differentiate into myocytic cells of myocardium. Our assumption was based on a previous report in which functional cardiomyocyte-like cells were obtained from mouse adipose tissue after their culturing in the medium containing SCF, IL-3 and IL-6 [10]. Similar cell culture scenario was also explored in case of human SVF both in our study and recently published analysis by Bai et al. [2].

In our experiments, the incubation of SVF in Methocult medium resulted in the expression of a few cardiac-specific transcription factors, such as GATA4 and MEF2C, which was accompanied by cells' morphological changes from fibroblast-like to cardiomyocyte-like shape [2].

The myocyte enhancer factor-2 (MEF2) proteins are transcription factors that are essential for differentiation process in all the muscle lineage cells. Cell-specific GATA transcription factors recruit MEF2 proteins to their target promoter sites. It is known, that inactivation of the MEF2C gene causes cardiac developmental arrest [13] and severe down-regulation of a number of early cardiac markers including ANP [7].

MYOD1 gene was also up-regulated in the cardiomyocyte-like cells obtained from the human SVF cells. This transcription factor is typically expressed in differentiated skeletal muscle cells, however there are reports that verify MyoD expression during the early steps of cardiomyocyte differentiation [8].
We postulate that incubation of SVF cells in Methocult medium in presence of SCF, IL-3 and IL-6 initiated cardiomyocyte phenotype differentiation only, since potentiation of expression of the cardiomyocyte progenitor cell marker ANP was not observed [11].

Acknowledgments This work was supported by F6 EU SC\&CR (LSHB-CT-2004-502988) project and Polish MNi/project Nr 2 PO5A 14230 .

Conflict of interest statement There is no conflict of interest.

\section{References}

1. Bacou F, el Andalousi RB, Daussin PA, Micallef JP, Levin JM, Chammas M, Casteilla L, Reyne Y, Nougues J (2004) Transplantation of adipose tissue-derived stromal cells increases mass and functional capacity of damaged skeletal muscle. Cell Transpl 13:103-111

2. Bai X, Pinkernell K, Song YH, Nabzdyk C, Reiser J, Alt E (2007) Genetically selected stem cells from human adipose tissue express cardiac markers. Biochem Biophys Res Commun 353:665-671

3. Balwierz A, Czech U, Polus A, Filipkowski RK, Mioduszewska B, Proszynski T, Kolodziejczyk P, Skrzeczynska-Moncznik J, Dudek W, Kaczmarek L, Kulig J, Pryjma J, Dembinska-Kiec A (2008) Human adipose tissue SVF cells differentiate to endothelial cells or adipocytes depending on distinct types of media. Cell Prol 41:441-459

4. Christoforou N, Gearhart JD (2007) Stem cells and their potential in cell-based cardiac therapies. Prog Cardiovasc Dis 49:396-413

5. Fraser JK, Schreiber RE, Zuk PA, Hedrick MH (2004) Adult stem cell therapy for the heart. Int J Biochem Cell Biol 36:658-666

6. Klein J, Perwitz N, Kraus D, Fasshauer M (2006) Adipose tissue as a source and target for novel therapies. Trends Endocr Metabol 17:26-32

7. Morin S, Charron F, Robitaille L, Nemer M (2000) GATAdependent recruitment of MEF2 proteins to target promoters. EMBO J 19:2046-2055

8. Olson EN (1993) Regulation of muscle transcription by the MyoD family. The heart of the matter. Circ Res 72:1-6

9. Pfaffl MW (2001) A new mathematical model for relative quantification in real-time PCR. Nucleic Acid Res 29:2002-2007

10. Planat-Benard V, Menard C, Andre M, Puceat M, Perez A, Garcia-Verdugo JM, Penicaud L, Casteilla L (2004) Spontaneous cardiomyocyte differentiation from adipose tissue stroma cells. Circ Res 94:223-229

11. Temsah R, Nemer M (2005) GATA factors and transcriptional regulation of cardiac natriuretic peptide genes. Regul Pept 128:177-185

12. Trayhurn P, Beattie JH (2001) Physiological role of adipose tissue: white adipose tissue as an endocrine and secretory organ. Proc Nutr Soc 60:329-339

13. Xu J, Gong NL, Bodi I, Aronow BJ, Backx PH, Molkentin JD (2006) Myocyte enhancer factors $2 \mathrm{~A}$ and $2 \mathrm{C}$ induce dilated cardiomyopathy in transgenic mice. J Biol Chem 281(14):91529162

14. Zuk PA, Zhu M, Ashjian P, De Ugarte DA, Huang JI, Mizuno H, Alfonso ZC, Fraser JK, Benhaim P, Hedrick MH (2002) Human adipose tissue is a source of multipotent stem cells. Mol Biol Cell 13:4279-4295 